\title{
Erosions in the foot at baseline are predictive of orthopaedic shoe use after 10 years of treat to target therapy in patients with recent onset rheumatoid arthritis
}

\author{
Sytske Anne Bergstra ${ }^{1}$ - Iris M. Markusse ${ }^{1} \cdot$ Gülşah Akdemir $^{1} \cdot$ H. Karel Ronday ${ }^{2}$. \\ K. Huub Han ${ }^{3}$ • Willem F. Lems ${ }^{4,5}$ • Pit J. S. M. Kerstens ${ }^{5}$ • Rosaline van den Berg ${ }^{1}$ • \\ Robert B. M. Landewé ${ }^{6,7}$ • Cornelia F. Allaart ${ }^{1}$
}

Received: 23 October 2015 /Revised: 25 November 2015 / Accepted: 6 December 2015 / Published online: 22 December 2015

(C) The Author(s) 2015. This article is published with open access at Springerlink.com

\begin{abstract}
The objective of this study is to investigate if foot joint damage due to rheumatoid arthritis (RA) can predict whether patients will start wearing orthopaedic shoes (OS) within 10 years after treatment start. Data from recent onset RA patients with 10 years follow-up from the BeSt (Dutch acronym for treatment strategies) study were used. Treatment was tightly controlled, targeted at disease activity score (DAS) $\leq 2.4$, according to 1 of 4 pre-specified treatment strategies. After 10 years of follow-up, orthopaedic shoe use was recorded in 285/508 patients (responders to questionnaires at 10 years). Between-group differences for orthopaedic shoe users and non-users were calculated at baseline, after 10 years, and change scores over the 10-year period were calculated. Predictors for orthopaedic shoe use were identified by
\end{abstract}

Electronic supplementary material The online version of this article (doi:10.1007/s10067-015-3145-1) contains supplementary material, which is available to authorized users.

Sytske Anne Bergstra

S.A.Bergstra@lumc.nl

1 Department of Rheumatology, Leiden University Medical Center, P.O. Box 9600, 2300 RC Leiden, The Netherlands

2 Department of Rheumatology, Haga-Leyenburg Teaching Hospital, The Hague, The Netherlands

3 Department of Rheumatology, Maasstad Hospital, Rotterdam, The Netherlands

4 Department of Rheumatology, VU Medical Center, Amsterdam, The Netherlands

5 Department of Rheumatology, Reade, Amsterdam, The Netherlands

6 Amsterdam Rheumatology \& Immunology Center, Academic Medical Center Amsterdam, Amsterdam, The Netherlands

7 Amsterdam Rheumatology \& Immunology Center, Atrium Medical Center Heerlen, Heerlen, The Netherlands univariable and multivariable logistic regression analyses. Orthopaedic shoe use was reported by 57/285 patients after 10 years. Orthopaedic shoe users had more joint damage, joint swelling and pain in the feet already at baseline and after 10 years. At both time points, DAS of orthopaedic shoe users and non-users was similar. Multivariable logistic regression showed that dichotomized foot erosions score (cut-off $\geq 1$ erosion) (OR 2.42), anti-citrullinated protein antibodies (ACPA) (OR 4.64) and DAS (OR 1.77) were independent predictors of orthopaedic shoe use. Despite intensive targeted treatment, 57/ 285 recent onset RA patients started using orthopaedic shoes over 10 year of follow-up. Presence of foot erosions at treatment start predicts orthopaedic shoe use after 10 years. The risk of orthopedic shoe use increased for ACPA-positive patients and for those with higher baseline disease activity.

Keywords Erosions · Orthopaedic shoes · Rheumatoid arthritis $\cdot$ Risk factors

\section{Introduction}

Despite major improvements in the treatment of rheumatoid arthritis (RA) patients, joint damage is still a common disease manifestation. The presence of erosions in an early disease stage is indicative of a potentially severe disease course with further joint damage progression $[1,2]$. Several authors have reported that in recent onset RA patients, erosions and joint space narrowing (JSN) occur more frequently in the feet than in the hands, particularly in the metatarsophalangeal joints [2-5].

Up to $80 \%$ of RA patients reported disease-related feet problems, often already in an early disease stage (53\% of patients) [6]. Reported symptoms include pain, stiffness, 
swelling and ultimately deformities of the foot joints with or without overlying ulcers [7, 8]. Symptoms of the feet may disturb the gait pattern, thereby negatively affecting mobility and health-related quality of life $[8,9]$.

It has been shown that wearing custom-made orthopaedic shoes (OS) is associated with pain reduction and improved mobility and activity participation $[10,11]$. Previously, 25 to $30 \%$ of RA patients with advanced RA reported OS use, which may underrepresent OS need, as many patients dislike OS $[12,13]$. Ideally, effective treatment of RA should prevent a need for OS. We hypothesized that in an intensely monitored, treated to target cohort of RA patients, such as participants of the BeSt study, the need for OS would be decreased compared to historic cohorts and investigated whether foot joint damage due to RA can predict OS use in patients who completed 10 years follow-up.

\section{Materials and methods}

\section{Patients}

Data from the BeSt (Dutch acronym for treatment strategies) study were used. The BeSt study has been described extensively before [14]. In short, it is a multicenter randomized trial (Dutch trial registry, NTR262 and NTR265) in which 508 recent onset RA patients (1987 American College of Rheumatology criteria [15]) were treated during a 10-year follow-up period. Patients were randomized into one of four treatment strategies: sequential monotherapy, step-up combination therapy, initial combination therapy with prednisone or initial combination therapy with infliximab. Treatment was adjusted if the three-monthly measured disease activity score in 44/53 joints (DAS) was $>2.4$. The Medical Ethical Committees of all participating centres approved both the study protocol and a separate protocol to approach all patients to fill out an additional questionnaire at 10 years. All patients gave written informed consent for the original study, and patients who filled out the questionnaire had signed a separate written informed consent.

At baseline and each following year, radiographs of the hands and feet were made. Two trained readers, unaware of the treatment strategy and patient data, have independently read the radiographs in random time order using the Sharp/ van der Heijde score (SHS) [16]. The final score was the two readers' mean score. At baseline, extensive disease measures [e.g., DAS, Health Assessment Questionnaire (HAQ) [17], erythrocyte sedimentation rate (ESR), Ritchie Articular Index (RAI), swollen joint count (SJC) in 66 joints] and patient and disease characteristics [e.g., IgM rheumatoid factor (RF), anti-citrullinated protein antibodies (ACPA), smoking status (yes/no) and body mass index (BMI)] were collected.
Every 3 months, disease activity measures and disease characteristics were obtained.

After 10 years follow-up, all original participants were asked by a questionnaire if they were currently using OS (either custom-made or off the shelf). Current use of OS, but not the time of first use, was recorded. Since all patients included in the BeSt study had recent onset RA (median 2 weeks from diagnosis to inclusion), it was assumed that none used OS at baseline.

\section{Statistical methods}

Patients using OS were compared to non-users regarding patient and disease characteristics at baseline and at year 10 . Change scores over the follow-up period were calculated and compared between OS users and non-users. Betweengroup differences for categorical variables were analysed using $\chi^{2}$ tests. Normally distributed variables were analysed using $t$ tests and non-normally distributed variables were analysed using Mann-Whitney $U$ tests. The four treatment strategies as well as mono- vs combination therapy groups were compared on the number of OS users ( $\chi^{2}$ tests). Logistic regression analyses were performed with OS use as binary outcome variable.

To avoid bias due to missing data in the logistic regression analyses, multiple imputation was performed for the predictor variables containing missing data (the continuous variables foot JSN and foot erosions and the dichotomous variable ACPA) using a multivariate normal model [18] with 50 imputation cycles.

In the logistic regression, a maximum of 1 predictor per 10 patients (outcome OS use) was modelled. First, univariable logistic regression was performed including baseline foot erosions and foot JSN as potential predictors. Since in the BeSt population, only few patients show severe radiographic damage, the distribution of the foot damage variables is highly skewed with many 'zeros' and relatively few high scores. Therefore, foot erosions and foot JSN were added as dichotomized predictors (cut-off value $\geq 1$ ), in order to better approximate this 'zero-inflated model'.

Predictors with a $p$ value $<0.1$ were added for further testing in a multivariable model. Next, age, BMI, smoking status, baseline DAS and a combination of ACPA and RF (four categories) were simultaneously added in a multivariable model, in combination with the contributory variables from the univariable regression. The selection of these variables was based on previous research [17-19]. The HAQ was not added as a predictor, due to its high correlation with the DAS.

Only predictors with statistically significant contribution were kept in the final model. Possible interaction between treatment arm and each predictor was tested in a logistic regression analysis with OS use as outcome variable. In case of statistically significant interaction $(p<0.05)$, OS use should be 
Table 1 Baseline characteristics for orthopaedic shoe users $(n=57)$ and non-users $(n=228)$

\begin{tabular}{|c|c|c|c|c|c|}
\hline & \multicolumn{2}{|c|}{ Orthopaedic shoe users } & \multicolumn{3}{|c|}{ Non-orthopaedic shoe users } \\
\hline & $N$ & & $N$ & & $p$ value \\
\hline Age years & 57 & $50.3(11.0)$ & 228 & $51.6(14.8)$ & 0.496 \\
\hline Female $(\%)$ & 57 & 68.4 & 228 & 67.1 & 0.877 \\
\hline BMI $\left(\mathrm{kg} / \mathrm{m}^{2}\right)$ & 57 & $26.4(3.3)$ & 228 & $26.0(3.8)$ & 0.503 \\
\hline Smoking (\% yes) & 57 & 38.6 & 228 & 29.8 & 0.567 \\
\hline Alcohol consumption ( $\%$ yes) & 57 & 56.1 & 227 & 51.3 & 0.181 \\
\hline Rheumatoid factor positive (\%) & 57 & 77.2 & 228 & 65.8 & 0.099 \\
\hline ACPA positive (\%) & 54 & 83.3 & 223 & 57.0 & $<0.001$ \\
\hline CRP [median (range)] & 57 & $20(0 ; 172)$ & 223 & $21(0 ; 237)$ & 0.895 \\
\hline DAS & 57 & $4.6(0.9)$ & 228 & $4.4(0.9)$ & 0.115 \\
\hline Ritchie articular index & 57 & $15.6(7.3)$ & 227 & $13.6(6.6)$ & 0.049 \\
\hline Total SJC & 57 & $15.3(7.2)$ & 227 & $14.5(6.3)$ & 0.385 \\
\hline TJC foot and ankle joints [median (range)] & 56 & $12(0 ; 26)$ & 226 & $10(0 ; 30)$ & 0.053 \\
\hline SJC foot and ankle joints [median (range)] & 56 & $6(0 ; 16)$ & 222 & $4(0 ; 21)$ & 0.033 \\
\hline ESR $[$ median (range) $]$ & 57 & $33(3 ; 97)$ & 228 & $36.5(2 ; 143)$ & 0.843 \\
\hline HAQ & 57 & $1.5(0.7)$ & 228 & $1.3(0.6)$ & 0.146 \\
\hline VAS pain (mm) & 57 & $56.2(18.4)$ & 227 & $53.4(22.2)$ & 0.381 \\
\hline VAS general health (mm) & 57 & $54.3(19.1)$ & 228 & $51.3(20.5)$ & 0.308 \\
\hline VAS disease activity (mm) & 57 & $61.5(19.8)$ & 227 & $59.7(23.2)$ & 0.581 \\
\hline VAS morning stiffness (mm) & 57 & $58.1(21.5)$ & 227 & $60.7(24.6)$ & 0.462 \\
\hline Patients with erosions (\%) & 55 & 40.4 & 222 & 24.1 & 0.017 \\
\hline Total SHS [median (range)] & 55 & $3(0 ; 21.5)$ & 213 & $1.5(0 ; 35.5)$ & 0.080 \\
\hline Bone erosions [median (range)] & 55 & $0(0 ; 14)$ & 213 & $0(0 ; 20.5)$ & 0.030 \\
\hline Bone erosions foot [median (range)] & 56 & $0(0 ; 14)$ & 215 & $0(0 ; 20.5)$ & 0.038 \\
\hline Bone erosions hands [median (range)] & 56 & $1(0 ; 3.5)$ & 218 & $0(0 ; 9.5)$ & 0.060 \\
\hline JSN [median (range)] & 55 & $2(0 ; 16.5)$ & 214 & $1.5(0 ; 19.5)$ & 0.193 \\
\hline JSN foot [median (range)] & 56 & $0(0 ; 8.5)$ & 217 & $0(0 ; 15)$ & 0.170 \\
\hline JSN hands [median (range)] & 56 & $1(0 ; 16.5)$ & 217 & $1(0 ; 34)$ & 0.025 \\
\hline
\end{tabular}

Mean (SD) reported if not stated otherwise

$A C P A$ anti-citrullinated protein antibodies, $B M I$ body mass index, $D A S$ disease activity score, $T J C$ tender joint count, SJC swollen joint count, ESR erythrocyte sedimentation rate, $H A Q$ health assessment questionnaire, vas visual analogue scale, CRP C-reactive protein, $S H S$ sharp/van der Heijde score, $J S N$ joint space narrowing analyzed per treatment arm. In case of no interaction, patients in all treatment arms were analyzed together. All analyses were performed using State SE version 14 (StataCorp LP).

\section{Results}

After 10 years, 307/508 patients (60\%) were still participating in the BeSt study. In total, 279/307 filled out the questionnaire about OS use at year 10. Patients had dropped out due to several reasons: 76 patients refused to continue participation, 9 had a revised diagnosis (no RA), 35 had a comorbidity contraindicating the pre-specified treatment, 42 had another reason (not further specified) and 39 died. Six patients who had dropped out agreed to fill out the questionnaire. In total, information about OS use after 10 years was available in 285/ 508 patients $(56 \%)$.

Responders were younger than non-responders (mean age 51.3 (SD 12.0) vs. 58.4 (SD 14.8) years, $p<0.001)$ and had a lower HAQ (mean 1.3 (SD 0.6) vs. 1.5 (SD 0.7), $p=0.020$ ) but did not differ statistically significantly on gender, smoking status, ACPA positivity, baseline DAS, total SHS, total JSN and total erosion scores (Supplementary Table 1).

Twenty percent of the responders $(57 / 285)$ reported to use OS. Baseline characteristics of OS users and non-users are presented in Table 1. OS users were statistically significantly more often ACPA positive and more frequently had erosions at baseline. They also had a statistically significantly higher total RAI, a higher RAI of the foot and ankle joints, a higher SJC of the foot and ankle joints and a higher total joint erosions score, foot erosions score and hand JSN score. The 
Table 2 Ten years data and change in patient and disease characteristics between baseline and 10 years for orthopaedic shoe users $(n=57)$ and non-users $(n=228)$

\begin{tabular}{|c|c|c|c|c|c|}
\hline \multirow[b]{3}{*}{ BMI $\left(\mathrm{kg} / \mathrm{m}^{2}\right)$} & \multirow{2}{*}{\multicolumn{2}{|c|}{$\begin{array}{l}\text { Orthopaedic shoe users } \\
N\end{array}$}} & \multirow{2}{*}{\multicolumn{2}{|c|}{$\begin{array}{l}\begin{array}{l}\text { Non-orthopaedic shoe } \\
\text { users }\end{array} \\
N\end{array}$}} & \multirow{3}{*}{$\begin{array}{l}p \text { value } \\
0.917\end{array}$} \\
\hline & & & & & \\
\hline & 56 & $26.7(4.3)$ & 208 & $26.6(4.2)$ & \\
\hline$\Delta \mathrm{BMI}\left(\mathrm{kg} / \mathrm{m}^{2}\right)$ & 56 & $0.2(0.3)$ & 208 & $0.6(2.5)$ & 0.304 \\
\hline Smoking ( $\%$ yes $)$ & 56 & 33.9 & 208 & 22.1 & 0.069 \\
\hline$\Delta$ Smoking & 56 & -4.8 & 208 & -7.7 & 0.936 \\
\hline Alcohol consumption (\% yes) & 55 & 60.0 & 207 & 63.0 & 0.685 \\
\hline$\Delta$ Alcohol consumption & 55 & 3.9 & 207 & 11.6 & 0.551 \\
\hline CRP [median (range)] & 55 & $3(1 ; 52)$ & 210 & $3(0 ; 147)$ & 0.752 \\
\hline$\triangle \mathrm{CRP}$ & 55 & $-15(-172 ; 36)$ & 205 & $-16(-231 ; 136)$ & 0.749 \\
\hline DAS & 55 & $1.7(0.8)$ & 216 & $1.6(0.7)$ & 0.440 \\
\hline$\Delta \mathrm{DAS}$ & 55 & $-2.9(1.1)$ & 216 & $-2.7(1.0)$ & 0.347 \\
\hline Ritchie articular index & 56 & $2.5(3.6)$ & 217 & $1.9(2.5)$ & 0.064 \\
\hline$\Delta$ Ritchie articular index & 56 & $-13.2(7.3)$ & 216 & $-11.5(6.4)$ & 0.097 \\
\hline Total SJC & 55 & $1.2(2.2)$ & 218 & $1.4(2.3)$ & 0.031 \\
\hline$\Delta$ Total SJC & 55 & $-14.3(7.4)$ & 217 & $-13.0(6.9)$ & 0.222 \\
\hline TJC foot/ankle joints [median (range)] & 56 & $12(0 ; 26)$ & 226 & $10(0 ; 30)$ & 0.053 \\
\hline$\Delta \mathrm{TJC}$ foot/ankle joints & 53 & $-8(-24 ; 46)$ & 210 & $-7(-30 ; 20)$ & 0.364 \\
\hline SJC foot/ankle joints [median (range)] & 56 & $6(0 ; 16)$ & 222 & $4(0 ; 21)$ & 0.033 \\
\hline$\Delta \mathrm{SJC}$ foot/ankle joints & 55 & $-5(-16 ; 0)$ & 212 & $-3.5(-21 ; 7)$ & 0.076 \\
\hline ESR [median (range)] & 54 & $18.4(17.4)$ & 217 & $20.1(18.0)$ & 0.337 \\
\hline$\Delta \mathrm{ESR}$ & 54 & $-20.5(-75 ; 31)$ & 217 & $-14(-105 ;-52)$ & 0.586 \\
\hline HAQ & 56 & $0.8(0.7)$ & 217 & $0.5(0.5)$ & 0.005 \\
\hline$\Delta \mathrm{HAQ}$ & 56 & $-0.7(0.7)$ & 217 & $-0.8(0.7)$ & 0.496 \\
\hline VAS pain (mm) & 56 & $23.7(23.6)$ & 216 & $19.6(20.9)$ & 0.205 \\
\hline$\Delta$ VAS pain & 56 & $-32.5(27.8)$ & 215 & $-33.4(25.1)$ & 0.809 \\
\hline VAS general health (mm) & 56 & $24.4(18.8)$ & 217 & $21.6(20.9)$ & 0.368 \\
\hline$\Delta$ VAS general health & 56 & $-30.59(26.4)$ & 217 & $-29.4(25.5)$ & 0.753 \\
\hline VAS disease activity (mm) & 56 & $24.3(22.3)$ & 217 & $20.3(21.1)$ & 0.223 \\
\hline$\Delta$ VAS disease activity & 56 & $-37.68(28.0)$ & 216 & $-39.0(27.3)$ & 0.741 \\
\hline VAS morning stiffness (mm) & 56 & $23.2(22.7)$ & 216 & $20.5(20.8)$ & 0.382 \\
\hline$\Delta$ VAS morning stiffness & 56 & $-34.3(27.3)$ & 215 & $-40.4(26.7)$ & 0.135 \\
\hline Patients with erosions (\%) & 55 & 75.4 & 225 & 58.8 & 0.020 \\
\hline$\Delta$ Patients with erosions & 55 & 35.1 & 225 & 34.7 & 0.593 \\
\hline Total SHS [median (range)] & 49 & $13.5(0 ; 146.5)$ & 206 & $6(0 ; 224.5)$ & 0.003 \\
\hline$\Delta$ Total SHS & 47 & $3.5(0.0 ; 145.5)$ & 193 & $2.00(-1.5 ; 215.5)$ & 0.007 \\
\hline Bone erosions & 49 & $4(0.0 ; 54.5)$ & 206 & $1(0 ; 98.5)$ & 0.008 \\
\hline$\Delta$ Bone erosions & 47 & $1.5(0.0 ; 50)$ & 192 & $0.05(-0.05 ; 97)$ & 0.009 \\
\hline Bone erosions foot [median (range)] & 51 & $1.5(0.0 ; 42.5)$ & 209 & $0(0 ; 32.5)$ & 0.002 \\
\hline$\Delta$ Bone erosions foot & 50 & $1.0(0.0 ; 28.5)$ & 197 & $0.0(-0.5 ; 31.0)$ & 0.002 \\
\hline Bone erosions hands [median (range)] & 51 & $0.5(0 ; 37.5)$ & 208 & $0(0 ; 66)$ & 0.061 \\
\hline$\Delta$ Bone erosions hands & 50 & $0.25(-0.05 ; 37.5)$ & 199 & $0.0(-0.5 ; 66)$ & 0.196 \\
\hline JSN [median (range)] & 50 & $8.25(0.0 ; 96.5)$ & 207 & $4(0 ; 126)$ & 0.004 \\
\hline$\Delta \mathrm{JSN}$ & 48 & $2.75(-1.5 ; 95.5)$ & 195 & $1.0(-1.5 ; 118.5)$ & 0.007 \\
\hline JSN foot [median (range)] & 52 & $2(0.0 ; 36)$ & 210 & $0(0 ; 40)$ & $<0.001$ \\
\hline$\Delta \mathrm{JSN}$ foot & 51 & $1.0(0.0 ; 36.0)$ & 200 & $0.0(0.0 ; 35.0)$ & $<0.001$ \\
\hline JSN hands [median (range)] & 51 & $4.5(0.0 ; 60.5)$ & 208 & $3(0 ; 86)$ & 0.025 \\
\hline$\Delta$ JSN hands & 50 & $1.25(-1.5 ; 59.5)$ & 199 & $0.0(-1.5 ; 83.5)$ & 0.037 \\
\hline
\end{tabular}

Mean (SD) reported if not stated otherwise

$R F$ rheumatoid factor, $A C P A$ anti-citrullinated protein antibodies, $B M I$ body mass index, $C R P$ c-reactive protein, $D A S$ disease activity score, TJC tender joint count, $S J C$ swollen joint count, $H A Q$ health assessment questionnaire, $V A S$ visual analogue scale, $S H S$ Sharp/van der Heijde score, $J S N$ joint space narrowing 
distribution of OS users and non-users over the allocated treatment groups did not significantly differ (Pearson $\chi^{2}(3)=6.6$, $p=0.085)$. Also, the proportion of patients in the arms grouped together as mono- or combination therapy did not significantly differ (Pearson $\chi^{2}(1)=2.03, p=0.154$ ).

In Table 2, patient and disease characteristics at year 10 as well as change scores over the 10-year follow-up period are shown. OS users more often had a higher HAQ, a higher SJC of the foot and ankle, as well as a higher total SHS and all of its sub-scores, except for hand erosions. The OS users also showed greater increase in total SHS and all of its sub-scores, except for hand erosions.

None of the predictors showed a statistically significant interaction with the allocated treatment group; therefore, all patients were analyzed together (data not shown). Presence of $\geq 1 \mathrm{ft}$ erosion (OR 2.32, $95 \%$ CI 1.13; 4.78) was a potential predictor in the univariable model (Table 3 ). In the multivariable model, presence of $\geq 1 \mathrm{ft}$ erosion, the combined ACPARF variable and DAS appeared statistically significant predictors of OS use. In the final model (Table 3, model 2), the combined ACPA-RF variable was the strongest predictor, with the highest OR for being ACPA+ but RF- (OR 6.14, $95 \%$ CI 1.68; 22.40) and a lower OR for being ACPA+ and $\mathrm{RF}+(\mathrm{OR} 4.90,95 \%$ CI 1.85; 12.99), followed by $\geq 1 \mathrm{ft}$ erosion (OR 2.33, $95 \%$ CI 1.08; 5.03) and baseline DAS (OR $1.77,95 \%$ CI $1.21 ; 2.60)$.

\section{Discussion}

This study showed that 57/285 (20\%) recent onset RA patients used OS after 10 years of tightly controlled treatment. This is less than in previous reports on patients with similar disease duration (25 to $30 \%$ ) [12, 13], yet higher than expected based on the tightly controlled treatment, with threemonthly joint evaluations including the feet, especially as it may be an underestimation of the actual need for OS [13]. OS in the Netherlands are partially reimbursed for all patients, and OS specialists are easily accessible, which may encourage prescription when medical therapy cannot alleviate symptoms. We investigated whether foot joint damage due to RA can predict OS use, with the underlying idea that with a better understanding of factors that associate with OS use, the need for OS may be prevented in future patients. To the best of our knowledge, this is the first study trying to predict OS use.

We found that OS users had more swollen joints and a trend towards more painful joints of feet and ankles both at baseline and at year 10. Also, joint damage at baseline and joint damage progression from baseline to year 10 was significantly greater in OS users for all damage sub-scores, except for hand erosions. There were no statistically significant differences in DAS (which includes the evaluation of feet and ankles) between the two groups at both time points, yet the DAS was slightly higher in OS users both at baseline and after 10 years.
Table 3 Logistic regression models to predict orthopaedic shoe use within 10 years of treatment start $(n=285)$

\begin{tabular}{lllll}
\hline Variable & Odds ratio & $\begin{array}{l}95 \% \text { confidence } \\
\text { interval }\end{array}$ & $p$ value & $\begin{array}{l}\text { Maximum } \\
\text { likelihood } R^{2}\end{array}$ \\
\hline Univariate predictors & & & & \\
Erosions foot yes/no & 2.32 & $1.13 ; 4.78$ & 0.022 & 0.017 \\
JSN foot yes/no & 1.42 & $0.75 ; 2.76$ & 0.281 & 0.004 \\
Multivariable models & & & & \\
Erosions foot yes/no & 2.42 & $1.10 ; 5.28$ & 0.027 & 0.10 \\
ACPA-RF & Ref. & Ref. & Ref. & \\
ACPA- RF+ & 1.10 & $0.25 ; 4.89$ & 0.896 & \\
ACPA+RF- & 6.43 & $1.75 ; 23.55$ & 0.005 & \\
ACPA+RF+ & 4.89 & $1.82 ; 13.13$ & 0.002 & \\
Age & 0.98 & $0.95 ; 1.01$ & 0.159 & \\
BMI & 1.05 & $0.96 ; 1.14$ & 0.296 & \\
DAS & 1.82 & $1.23 ; 2.67$ & 0.002 & \\
Smoking status yes/no & 1.29 & $0.66 ; 2.51$ & 0.453 & \\
Erosions foot yes/no & 2.33 & $1.08 ; 5.03$ & 0.031 & \\
ACPA-RF & Ref. & Ref. & Ref. & \\
ACPA- RF+ & 1.12 & $0.26 ; 4.93$ & 0.876 & \\
ACPA+RF- & 6.14 & $1.68 ; 22.40$ & 0.006 & \\
ACPA+RF+ & 4.90 & $1.85 ; 12.99$ & 0.001 & \\
DAS & 1.77 & $1.21 ; 2.60$ & 0.003 & \\
& & & &
\end{tabular}

For all binary variables, 'no' was used as the reference category. For ACPA-RF, 'both negative' was used as the reference category

$J S N$ joint space narrowing, $A C P A$ anti-citrullinated protein antibodies, $D A S$ disease activity score. 
The HAQ of OS users and non-users was comparable at baseline, but after 10 years, a statistically significant and clinically meaningful difference of 0.23 in HAQ score was found between OS users and non-users, with OS users reporting lower functional ability than non-users, despite having similar disease activity. We found that the presence of at least one baseline foot erosion, ACPA positivity with or without RF positivity and a higher DAS at treatment start were independent predictors of OS use after 10 years. RF positivity without ACPA positivity, higher age, BMI, smoking status and foot JSN at baseline were not predictive of future OS use.

This study has some limitations. The date of onset of OS use is unknown, limiting our analysis to potential baseline predictors, in a relatively homogenous patient cohort, due to study inclusion criteria. Nevertheless, it is unlikely that OS were the cause of joint destruction and functional disability $[10,19,20]$.

Another limitation of our study is the low response to the 10 years questionnaire. Although most baseline characteristics did not differ between questionnaire responders and non-responders, we do not know what happened with nonresponders regarding OS use. Since questionnaire responders were slightly older than non-responders, younger patients appear to be underrepresented, but how this would affect the outcomes is unknown. The low number of OS users $(n=57)$ allowed us to include only a limited number of potential predictors in the logistic regression analysis. Nevertheless, we have found some predictors of OS use that were in line with previously reported data about radiographic damage progression [21].

In conclusion, after 10 years of DAS $\leq 2.4$ targeted treatment, 57/285 (20\%) recent onset RA patients reported to use OS. Presence of foot erosions at treatment start is associated with OS use after 10 years, with ACPA positivity with or without RF positivity and — to a lesser extent—a high baseline DAS contribute additional risk.

Acknowledgments We would like to thank all patients for their contribution, as well as the following rheumatologists who participated in the BeSt Study Group: J. van Aken, H. Boom and M. F. van Lieshout-Zuidema (Spaarne Hospital, Hoofddorp); W. M. de Beus, M. H. W. de Bois, M. de Buck, G. Collée and L. Lard (Medical Center Haaglanden, Leidschendam); C. Bijkerk and A. J. Peeters (Reinier de Graaf Gasthuis, Delft); B. A. C. Dijkmans, J. A. P. M. Ewals, H. van der Leeden, A. Linssen, P. E. H. Seys and J. Ph. Terwiel (retired); F. Fodili, J. H. L. M. van Groenendael and J. B. Harbers (Fransiscus Hospital, Roosendaal); A. H. Gerards and P. A. H. M. van der Lubbe (Vlietland Hospital, Schiedam); R. J. Goekoop, Y. P. M. Goekoop-Ruiterman, N. Riyazi and A. A. Schouffoer (Haga Hospital, The Hague); B. A. M. Grillet (Zorgsaam, Terneuzen); A. L. Huidekoper, I. Speyer, G. M. Steup-Beekman and M. L. Westedt (Bronovo Hospital, The Hague); M. V. van Krugten (Admiraal de Ruyter Hospital, Vlissingen); M. C. Lodder, C. Mallée, K. S. S. Steen and S. ten Wolde (Kennemer Gasthuis, Haarlem); E. T. H. Molenaar and M. van Oosterhout (Groene Hart Hospital, Gouda); D. van Schaardenburg and A.E. Voskuyl (VU Medical Center, Amsterdam); P. B. J. de Sonnaville (Admiraal de Ruyter Hospital, Goes); and D. van Zeben (Sint Franciscus Gasthuis, Rotterdam).
We would also like to thank all other rheumatologists and trainee rheumatologists who enrolled patients in the BeSt Study, as well as all research nurses for their contributions.

Compliance with ethical standards The medical ethical committees of all participating centres approved both the study protocol and a separate protocol to approach all patients to fill out an additional questionnaire at 10 years. All patients gave written informed consent for the original study, and patients who filled out the questionnaire had signed a separate written informed consent.

\section{Disclosures None.}

Funding The study was designed by the investigators and supported by a government grant from the Dutch College of Health Insurance Companies, with additional funding from Schering-Plough B.V. and Janssen B.V.. Data collection, trial management, data analysis and preparation of the manuscript were performed by the authors.

Open Access This article is distributed under the terms of the Creative Commons Attribution 4.0 International License (http:// creativecommons.org/licenses/by/4.0/), which permits unrestricted use, distribution, and reproduction in any medium, provided you give appropriate credit to the original author(s) and the source, provide a link to the Creative Commons license, and indicate if changes were made.

\section{References}

1. van Nies JA, van Steenbergen HW, Krabben A et al. (2014) Evaluating processes underlying the predictive value of baseline erosions for future radiological damage in early rheumatoid arthritis. Ann Rheum Dis 74:883-889

2. van der Heijde DM, van Leeuwen MA, van Riel PL et al (1992) Biannual radiographic assessments of hands and feet in a three-year prospective followup of patients with early rheumatoid arthritis. Arthritis Rheum 35:26-34

3. Mottonen TT (1988) Prediction of erosiveness and rate of development of new erosions in early rheumatoid arthritis. Ann Rheum Dis 47:648-53

4. van der Heijde DM (1995) Joint erosions and patients with early rheumatoid arthritis. Br J Rheumatol 34(Suppl 2):74-8

5. Landewe RB, Strand V, Conaghan PG, van der Heijde D (2011) Damage and progression on radiographs in individual joints: data from pivotal randomized controlled trials. J Rheumatol 38:2018-22

6. Grondal L, Tengstrand B, Nordmark B, Wretenberg P, Stark A (2008) The foot: still the most important reason for walking incapacity in rheumatoid arthritis: distribution of symptomatic joints in 1,000 RA patients. Acta Orthop 79:257-61

7. van der Leeden M, Steultjens MP, Ursum J et al (2008) Prevalence and course of forefoot impairments and walking disability in the first eight years of rheumatoid arthritis. Arthritis Rheum 59:1596602

8. Laroche D, Ornetti P, Thomas E, Ballay Y, Maillefert JF, Pozzo T (2007) Kinematic adaptation of locomotor pattern in rheumatoid arthritis patients with forefoot impairment. Exp Brain Res 176: 85-97

9. Wickman AM, Pinzur MS, Kadanoff R, Juknelis D (2004) Healthrelated quality of life for patients with rheumatoid arthritis foot involvement. Foot Ankle Int 25:19-26

10. Dahmen R, Buijsmann S, Siemonsma PC, Boers M, Lankhorst GJ, Roorda LD (2014) Use and effects of custom-made therapeutic footwear on lower-extremity-related pain and activity limitations 
in patients with rheumatoid arthritis: a prospective observational study of a cohort. J Rehabil Med 46:561-7

11. Farrow SJ, Kingsley GH, Scott DL (2005) Interventions for foot disease in rheumatoid arthritis: a systematic review. Arthritis Rheum 53:593-602

12. Matricali GA, Boonen A, Verduyckt J et al (2006) The presence of forefoot problems and the role of surgery in patients with rheumatoid arthritis. Ann Rheum Dis 65:1254-5

13. de Boer IG, Peeters AJ, Ronday HK, Mertens BJ, Huizinga TW, Vliet Vlieland TP (2009) Assistive devices: usage in patients with rheumatoid arthritis. Clin Rheumatol 28:119-28

14. Goekoop-Ruiterman YP, de Vries-Bouwstra JK, Allaart CF et al (2005) Clinical and radiographic outcomes of four different treatment strategies in patients with early rheumatoid arthritis (the BeSt study): a randomized, controlled trial. Arthritis Rheum 52:3381-90

15. Arnett FC, Edworthy SM, Bloch DA et al (1988) The American rheumatism association 1987 revised criteria for the classification of rheumatoid arthritis. Arthritis Rheum 31:315-24
16. van der Heijde D (1999) How to read radiographs according to the Sharp/van der Heijde method. J Rheumatol 26:743-5

17. Siegert CE, Vleming LJ, Vandenbroucke JP, Cats A (1984) Measurement of disability in Dutch rheumatoid arthritis patients. Clin Rheumatol 3:305-9

18. Little RJA, Rubin DB (2002) Statistical analysis with missing data, Second Edition. Hoboken, New Jersey

19. Hennessy K, Burns J, Penkala S (2007) Reducing plantar pressure in rheumatoid arthritis: a comparison of running versus off-the-shelf orthopaedic footwear. Clin Biomech (Bristol, Avon) 22:917-23

20. Riskowski J, Dufour AB, Hannan MT (2011) Arthritis, foot pain \& shoe wear: current musculoskeletal research on feet. Curr Opin Rheumatol 23:148-55

21. Visser K, Goekoop-Ruiterman YP, de Vries-Bouwstra JK et al (2010) A matrix risk model for the prediction of rapid radiographic progression in patients with rheumatoid arthritis receiving different dynamic treatment strategies: post hoc analyses from the BeSt study. Ann Rheum Dis 69:1333-7 